\title{
Pathological Investigations And Correlation Research of Microfibrillar-Associated Protein 4 And Tropoelastin In Oral Submucous Fibrosis
}

Binjie Liu

Central South University

Wenqun Gou

Central South University

Hui Feng ( $\sim$ fengh0929@163.com)

Central South University

\section{Research Article}

Keywords: Microfibrillar associated protein 4, tropoelastin, oral submucous fibrosis, Immunohistochemistry Pathological Characteristic

Posted Date: September 13th, 2021

DOI: https://doi.org/10.21203/rs.3.rs-842918/v1

License: (9) This work is licensed under a Creative Commons Attribution 4.0 International License. Read Full License

Version of Record: A version of this preprint was published at BMC Oral Health on November 19th, 2021. See the published version at https://doi.org/10.1186/s12903-021-01962-w. 


\title{
Pathological Investigations and Correlation Research of Microfibrillar-associated protein 4 and tropoelastin in Oral Submucous Fibrosis
}

\author{
Binjie Liu ${ }^{1 \dagger}$, Wenqun Gou ${ }^{1,2 \dagger}$, Hui Feng ${ }^{1}$ \\ ${ }^{1}$ Department of Oral Medicine, Xiangya Stomalogical Hospital, Central South University, \\ Changsha, China \\ ${ }^{2}$ Changsha Stomatological Hospital, Changsha, China \\ ${ }^{\dagger}$ These authors contributed equally to this work.
}

CONFLICTS OF INTEREST: None to declare.

Correspondence

Hui Feng - Department of Oral Medicine, Xiangya Stomalogical Hospital, Central South University, Changsha, China. Email: fengh0929@163.com

\begin{abstract}
Background: Oral submucous fibrosis (OSF), distinguished by abnormal collagen deposition, is a precancerous disorder with $7 \%-30 \%$ of malignant transformation and rising global prevalence. However, the precise pathogenesis and effective treatment still remains elusive and controversial despite superfluity of literature. Therefore, it is extremely necessary and significant to explore the clinicopathological characteristics and potential markers for diagnosis and prognosis of OSF. Here, the objective of this research is to evaluate the influence and correlation of Microfibrillar-associated protein 4 [MFAP4] and tropoelastin [TE] on the development of OSF patients.
\end{abstract}

Material and Methods: Classic clinicopathological factors, HE and Masson trichome staining, immunohistochemical characteristics and the correlation (MFAP4 and TE) were recorded and compared among different stages of OSF cases $(n=60)$ and among those normal individuals $(\mathrm{n}=10)$. Then, the comparison using Student's $\mathrm{t}$ test, ANOVA analysis, the chi-square test for categorical variables was conducted in clinicopathological characteristics and the expression level of MFAP4 and TE between the patients' and normal tissue. The correlation analysis of MFAP4 and TE were assessed via means of Pearson's correlation test and linear regression.

Results: MFAP4 and TE proteins are upregulated and even increasing gradually in varying grades of OSF patients relative to the normal cases. Furthermore, statistical analyses yielded that the expression level of MFAP4 was positively associated with TE, and the Pearson correlation coefficient was $0.3781(\mathrm{p}=0.0048)$. Clinically, we found that OSF affected more male than female with a ratio of 29: 1 . The age range was 16-60 years, and the mean age was $36.25 \pm 10.25$ years old. Moreover, the positive expression rate of MFAP4 and TE in patients less than 40 years old is higher than that of those over 40 years old. Meanwhile, all OSF cases had chewed areca nut, with $51.67 \%$ smoking tobacco. 
Conclusions: Our study elucidates that the accumulation of MFAP4 and TE proteins may play a vitally important effect in the occurrence and development of OSF and has a hope to become a promising candidate molecular for prevention, diagnosis, and treatment strategies of OSF in the future.

Keywords: Microfibrillar-associated protein 4, tropoelastin, oral submucous fibrosis, Immunohistochemistry; Pathological Characteristic

\section{Introduction}

Oral Submucous Fibrosis (OSF) is a chronic, irreversible, persistent progressive, potentially malignant disease with the malignant transformation rate of $7 \%-30 \%$ [1-3]. Currently, the extremely significant accumulation of the extracellular matrix (ECM) both in OSF and other fibrotic diseases has attracted proverbially attention. The defective ECM dynamics stimulating by arecoline leads to the declined of collagen clearance and increased of collagen synthesis in the development of fibrosis diseases [4]. Inspired by this, our previous preliminary findings have observed that MFAP4, an extracellular matrix protein, was notably upregulated in OSF tissues by proteomic analysis [5]. MFAP4 is a ubiquitous protein playing an increasingly noteworthy part in elastin fiber formation and ECM remodeling processes during vascular injury and multitudinous fibrotic diseases, including myocardium, liver, joint and renal fibrosis [6-12]. In viral hepatitis and cirrhosis patients, MFAP4 levels increased significantly from non-fibrosis stage to the severe stage via transcription and protein levels experiment and histochemical analysis [13, 14]. Meanwhile, extensive research has shown that serum MFAP4 level can be used as a diagnosis predictor of varying degrees of liver cirrhosis and liver fibrosis [15]. Additionally, the finding revealed that plasma and atrial MFAP4 protein expression were elevated in atrial fibrosis rats [16]. Although MFAP4 is a crucial ECM protein in development of various fibrotic diseases, the expression and distribution of MFAP4 in various stages of OSF is not yet fully elucidated.

Interestingly, MFAP4 protein is capable of binding specifically to TE protein and further actively promotes TE self-assembly [17]. TE, also an extracellular matrix protein, is a soluble precursor of elastin and principal structural constituent of microfibrils in elastic fibers. And TE protein is excreted by fibroblasts and vascular smooth muscle cells (VSMC) $[18,19]$. Moreover, up-regulated TE protein is involved in the process of fibrosis through influencing on fibroblasts, inflammatory cells and angiogenesis in skin, lung and liver fibrosis [20-22], $[14,23]$. However, the expression of TE protein and the correlation between MFAP4 and TE in the development of OSF remains unknown.

Therefore, in this study, appropriate samples with definite diagnosis were selected by H\&E staining and Masson staining; and then the changes of MFAP4 and TE in OSF patients were detected by immunohistochemistry. Finally, the clinical information of 60 patients was analyzed retrospectively and statistically. Given the above, the purpose of this study was to visualize the presence and correlation of MFAP4 and TE involving in the progression of OSF, and to hope the two proteins for becoming a promising candidate for biomarkers and therapeutic targets in OSF patients in the future. 


\section{Materials and Methods}

\section{1 sample selection}

All clinical samples and clinical data were collected from patients of the Department of Maxillofacial Surgery, Periodontology and Pathology, Xiangya Stomatological Hospital and Xiangya Hospital, Central South University, Hunan, China. Samples were collected from 2014 to 2020. Informed consent was signed by all study participants, and the research was authorized by the institutional research ethics committee of Xiangya Stomatological Medical College (approval number 20200034). A total of 10 young Chinese (no betel quid chewing and smoking) without current or prior oral mucosal diseases provided the control samples. Different stage of OSF individuals was identical with relevant clinical manifestation and pathological characteristics. The OSF cases were graded according to Wollina, U and Verma, S. B. staging [24]. The diagnoses were assessed and independently verified by two experienced pathologists of Xiangya Stomatological Hospital and Xiangya Hospital.

\subsection{H\&E staining and Masson staining}

H\&E staining was conducted according to the standard procedure and was applied to observe cell morphological change of normal oral mucosa and OSF tissue. Masson staining via a classic three-color method was used to check and define the pathological phase of the OSF specimen, and observe the distribution of collagen under the microscope. The slides store at room temperature.

\subsection{Immunohistochemistry}

The tissue samples were collected and were fixed with paraformaldehyde for $48 \mathrm{~h}$, embedded in paraffin, then sectioned at 4- $\mu$ m-thick for immunohistochemical reaction (IHC). After deparaffinization and rehydration, the tissue sections were subjected to antigen retrieval in 10 $\mathrm{mM}$ sodium citrate solution $(\mathrm{pH}$ 6.0). While the antigen retrieval solution freely cooled to indoor temperature, the samples were then blocked with 3\% hydrogen peroxide to interdict endogenous peroxidase. Primary antibodies were diluted with 3\% BSA solution at the appropriated ratios: MFAP4 (ab103925; Abcam), 1: 500 and TE (ab21600; Abcam), $1: 500$. In a humidified box, the histologic sections incubated with the labeled primary antibody stay overnight at $4{ }^{\circ} \mathrm{C}$. The second day, the applicable secondary antibodies incubated for $30 \mathrm{~min}$ followed by three PBS buffer washes for five minutes each followed for incubation with a fresh solution of 2,3-diaminobenzidine (DAB) hydrochloride substrate.

The stained slides were allocated in a double-blind form and assessed under the light microscope to eliminate inter-observer bias. Immuno-staining score was measured by the following two rules: 1$)$ the staining intensity corresponding to score 1 ( 0 , negative; 1 , weak; 2 , moderate; 3 , strong). 2$)$ the range of staining corresponding to score $2(0,<5 \% ; 1,6 \%-25 \% ; 2$, $26 \%-50 \% ; 3,51 \%-75 \% ; 4,>75 \%$ ). The total score of the staining intensity was multiplying score 1 by score 2 and was applied to statistical analysis.

All methods were carried out in accordance with relevant guidelines and regulations.

\section{4 statistical analysis}

The data were evaluated using GraphPad Prism 8. Student's t test and ANOVA analysis were 
applied to assess the expression of MFAP4 and TE proteins in all tissue samples. The comparison using the chi-square test for categorical variables was conducted in clinicopathological characteristics of the sick and the expression of MFAP4 and TE between the patients' and normal tissue. The correlations between MFAP4 and TE were assessed via means of Pearson's correlation test and linear regression. For all experiments, significance was evaluated as *, p $<0.05 ; * *, \mathrm{p}<0.01$; **, $\mathrm{p}<0.001$; and $\mathrm{ns}>0.05$.

\section{Results}

\subsection{Clinical specimen Classification and Pathological Features Evaluation}

10 normal mucosal and 60 OSF samples were collected and OSF tissues (13 early, 26 middle, and 21 late OSF tissues) were classified by H\&E staining and Masson staining. As shown in Figure $1 \mathrm{a}, \mathrm{b}$, in the early phase, the epithelial layer was no significant change compared to normal tissues. Nevertheless, some fine collagen fibers maintaining its original structure were presented and were properly looser in the juxta-epithelial area. In the meantime, the majority of the blood vessels were normal, sometimes dilated and congested and some lesion manifested features of slight inflammation in the sub-epithelial tissue. In the mid-stage of OSF, the lesion area displayed part of hyaline degeneration, collagen fiber in the lamina propria was strip-like or banded, and even lumen diameter of the vessels decreased obviously (Figure $1 \mathrm{c}$ ). In the late stage of fibrosis, epithelial atrophy can be seen in the epithelial layer, and vacuolar degeneration occurs in each layer of the epithelium, especially in spinal cell layer. All fibrous collagen was glass-like, the normal structure disappeared completely, and the blood vessels were narrowed or occluded (Figure $1 \mathrm{~d}$ ).

(a) Normal

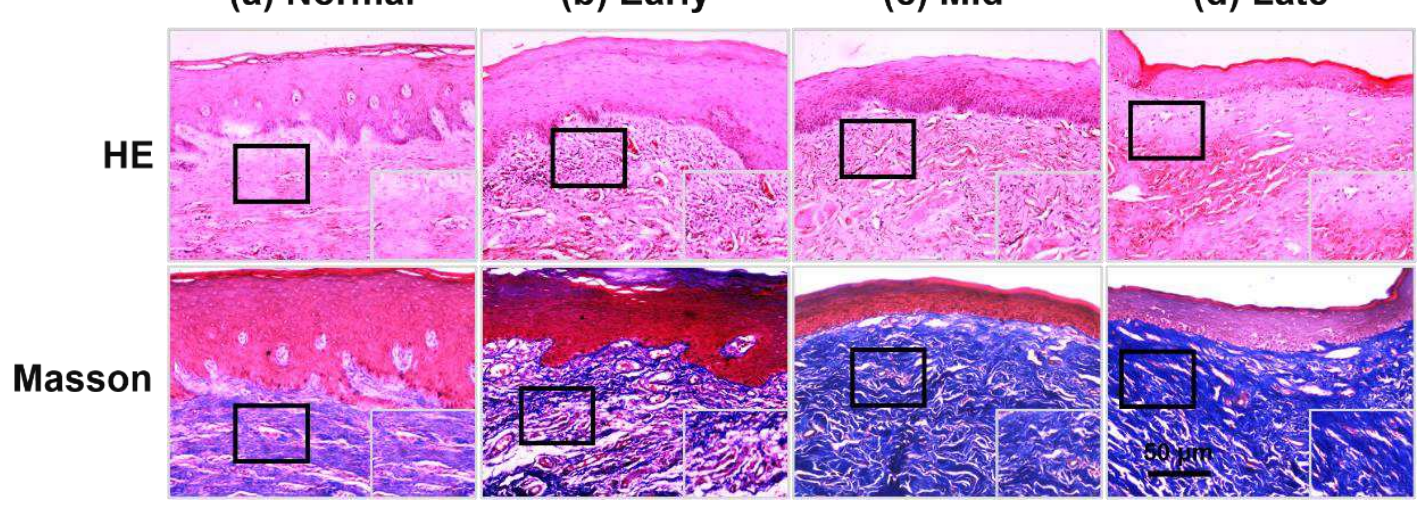

Fig. 1 HE staining and Masson staining. (a) The structure of normal oral mucosa tissue. (b) Mild inflammatory infiltrates of juxta-epithelial area and blood vessels in the early phase of OSF. (c) Limited hyaline degeneration, obvious collagen accumulation with stripy or banded fibers, and significantly reduced lumen size in the mid stage of OSF. (d) Visibly atrophied epithelium, epithelial vacuolar degeneration, plate-like collagen fibers, and diffused hyaline degeneration leading to narrowed or occluded vascula in the late stage of OSF.

\subsection{The excessive expression of MFAP4 and TE proteins is involved in the progressing of OSF}

To elucidate the relationship between ECM protein MFAP4 expression and OSF progression, 
clinical OSF samples at different stages were performed by immunohistochemistry. Originally, we observe the collagen deposition in various pathological stages of OSF via using Masson staining. The findings indicated that more intense blue staining in the submucosa from the collagen deposition in all OSF tissues compared with that of normal tissues. And as the degree of fibrosis increases gradually, blue staining in the connective tissue exists increasingly stronger intensity (Figure 1). Afterwards, we discovered that strong MFAP4 immunoreactivity was identified in subepithelial connective tissues of these OSF tissues in different stages ( $\mathrm{n}=70, \mathrm{P}=0.0007$ ) (Figure 2, $3 \mathrm{a}$ ). Moreover, the expression level of MFAP4 protein was slightly increased in the early-stage $(n=13, p=0.0015)$ and mid-stage $(n=26, p$ $=0.0001)$ and significantly increased in the late-stage $(n=21, p<0.0001)$.

In previous research, interestingly, we found that TE and MFAP4 participate jointly in elastin assembly and are closely related to the progression of plenty of fibrotic diseases [14, 17, 21, 25]. However, it is worth noting that the expression of TE protein at different OSF stages is unexplored. So we performed the expression and distribution of TE protein in the disease via immunohistochemical staining methods. Our results showed that the tendency of remarkably increasing intensity of TE was observed in submucosa area from normal tissue, early stage (n $=13, \mathrm{P}=0.0010)$ to mid- $(\mathrm{n}=26, \mathrm{p}<0.0001)$ and late-stage tissues $(\mathrm{n}=21, \mathrm{p}<0.0001)$, being consistent with the expression pattern of MFAP4, as illustrated in Figure 2 and Figure 3 $\mathrm{b}(\mathrm{n}=70, \mathrm{P}<0.0001)$. Meanwhile, MFAP4 and TE proteins were occasionally expressed in blood vessels.

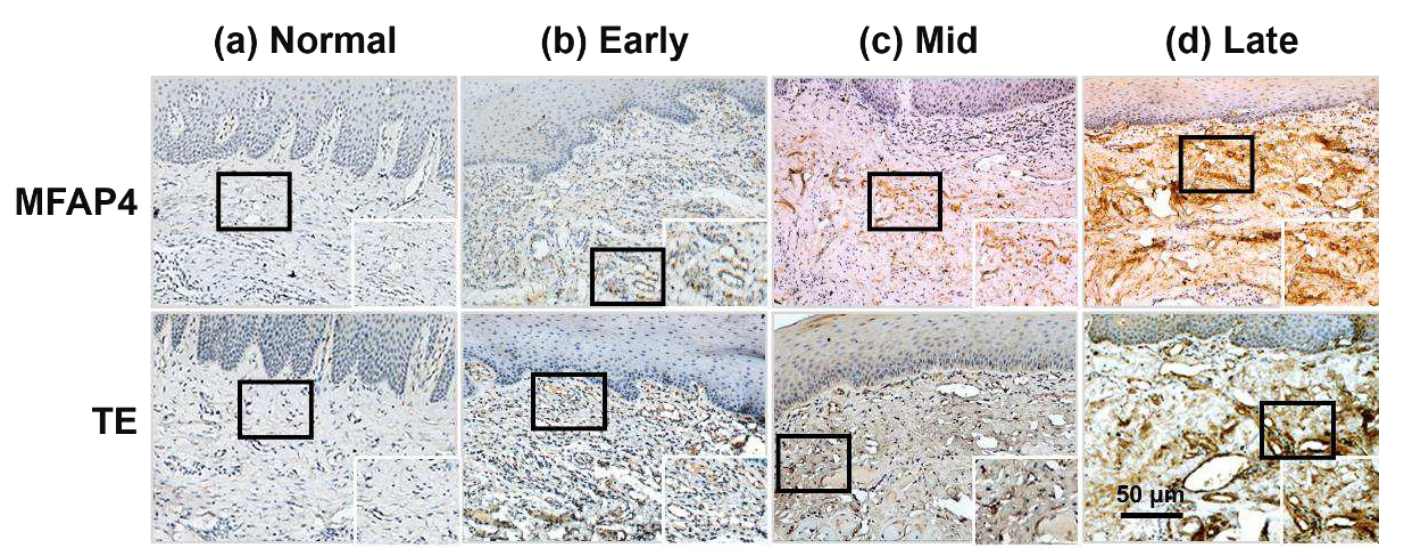

Fig. 2 Immunohistochemistry analysis of MFAP4 and TE in normal oral mucosa and OSF tissues. IHC images showing the incremental expression of MFAP4 and TE in the normal(a), early(b), middle(c) and late stages(d) of OSF.

In order to investigate the potential correlations of MFAP4 and TE protein expression in OSF tissues, Pearson correlation test was devoted to establish the correlation model. Results from statistical analyses yielded that the expression level of MFAP4 was positively associated with $\mathrm{TE}$, and the Pearson correlation coefficient is $0.3781(\mathrm{p}=0.0048)$ (Figure $3 \mathrm{c}$ ). Briefly, there is a significant positive correlation between MFAP4 and TE expression in the OSF submucosa area, suggesting that they have a close relationship with the progression of OSF. 
(a)
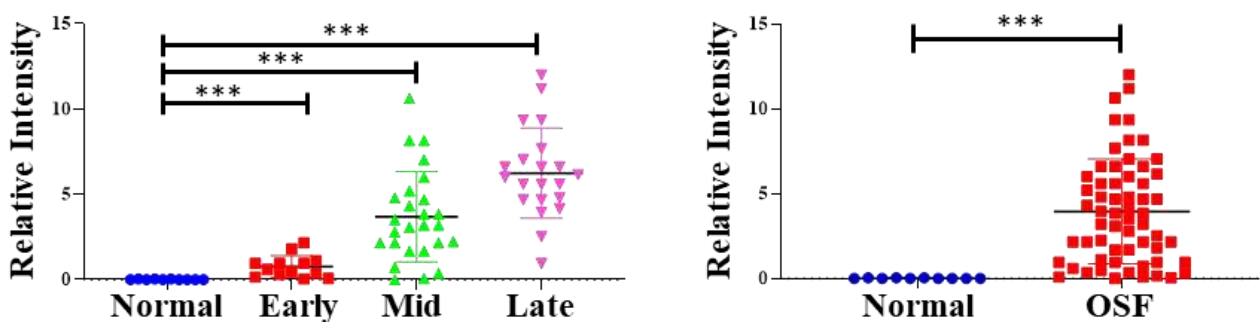

(b)

TE

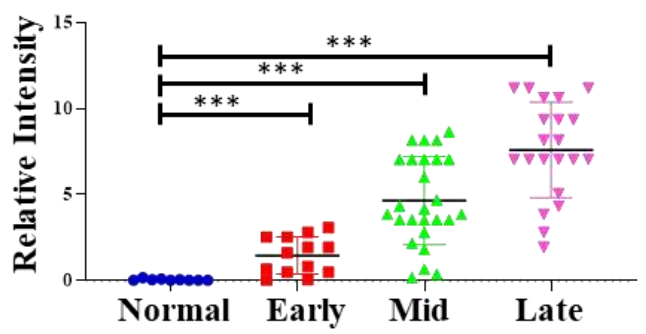

TE
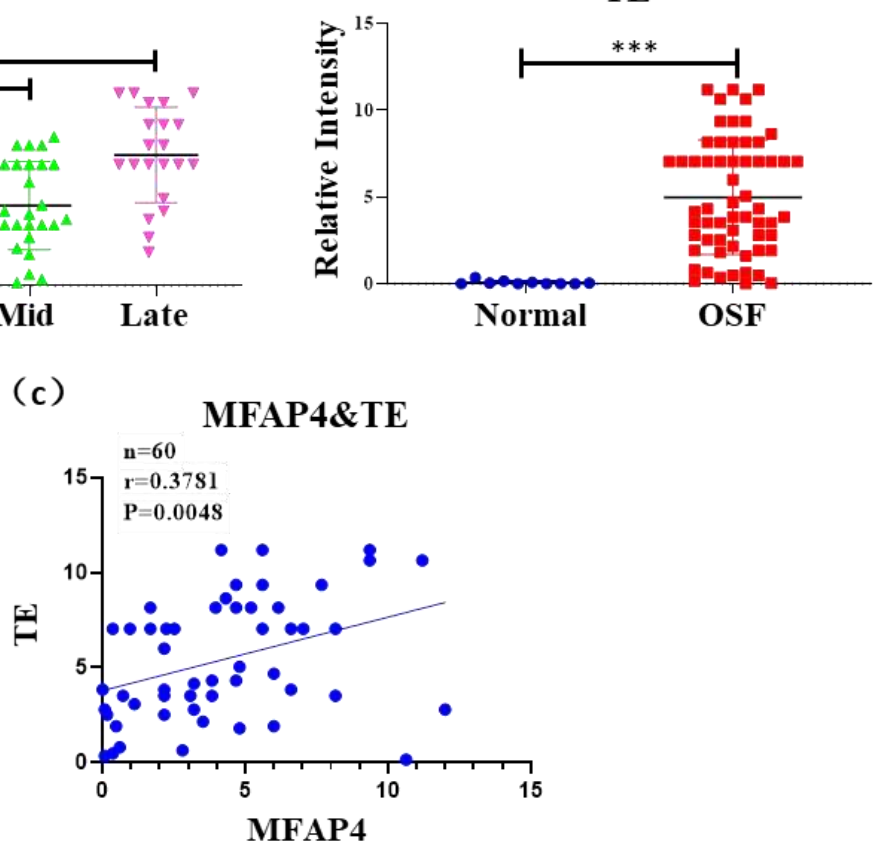

Fig. 3 Statistical results of the IHC intensity scores (a b) and correlation analysis (c) displaying the positively correlation of MFAP4 and TE in normal oral mucosa and different stages of OSF tissues. *: $\mathrm{p}<0.05, * *$ : $p<0.01, * * *: \mathrm{p}<0.001$.

\subsection{Clinicopathological features of OSF}

To investigate the clinicopathological features, the clinical information of 60 OSF patients were obtained. We found that OSF affects more male than female with a ratio of 29: 1 . The age range was 16-60 years, and the mean age was $36.25 \pm 10.25$ years old. OSF was most frequent in the age range of 31- 40 years (36.67\%). Consistently, the positive expression rate of MFAP4 and TE in patients less than 40 years old is higher than that of those over 40 years old. Of the OSF cases, all had chewed areca nut, with $51.67 \%$ smoking tobacco (TABLE 1). 
TABLE 1 The Evaluation of Clinicopathological features associated with the expression of MFAP4 and TE of OSF patients

\begin{tabular}{|c|c|c|c|c|c|c|c|}
\hline \multirow[b]{2}{*}{ Characteristics } & \multirow{2}{*}{$\begin{array}{l}\text { No of } \\
\text { sample } \\
(\%)\end{array}$} & \multicolumn{2}{|c|}{ MFAP4 } & \multirow[b]{2}{*}{ P Value } & \multicolumn{2}{|c|}{$\mathrm{TE}$} & \multirow[b]{2}{*}{ P Value } \\
\hline & & $\begin{array}{l}\text { Negativ } \\
\mathrm{e}\end{array}$ & Positive & & $\begin{array}{l}\text { Negativ } \\
\mathrm{e}\end{array}$ & Positive & \\
\hline \multicolumn{8}{|l|}{ Sex(patients) } \\
\hline Male & $58(96.7)$ & 15 & 43 & $\mathrm{~ns}$ & 8 & 50 & $\mathrm{~ns}$ \\
\hline Female & $2(0.03)$ & 0 & 2 & & 1 & 1 & \\
\hline \multicolumn{8}{|l|}{ Age (patients) } \\
\hline$<40$ & $42(70)$ & 7 & 35 & $0.0475 *$ & 3 & 39 & $0.0164 *$ \\
\hline$\geq 40$ & $18(30)$ & 8 & 10 & & 6 & 12 & \\
\hline \multicolumn{8}{|l|}{ Stage } \\
\hline Normal & $10(14.3)$ & 10 & 0 & $\begin{array}{l}<0.0001 \\
* * *\end{array}$ & 10 & 0 & $\begin{array}{l}<0.0001 \\
* * *\end{array}$ \\
\hline Early & 13(18.6) & 10 & 3 & & 6 & 7 & \\
\hline Mid & $26(37.1)$ & 4 & 22 & & 3 & 23 & \\
\hline Late & $21(30)$ & 1 & 20 & & 0 & 21 & \\
\hline \multicolumn{8}{|l|}{$\begin{array}{l}\text { Betel quid } \\
\text { chewing }\end{array}$} \\
\hline Yes & $60(100)$ & 19 & 41 & ns & 15 & 45 & ns \\
\hline No & $0(0)$ & 0 & 0 & & 0 & & \\
\hline \multicolumn{8}{|l|}{ Smoking } \\
\hline Yes & $31(51.7)$ & 10 & 21 & 0.2377 & 6 & 25 & 0.4743 \\
\hline No & $29(48.3)$ & 5 & 24 & & 3 & 26 & \\
\hline \multicolumn{8}{|l|}{$\begin{array}{l}\text { Clinical } \\
\text { feature }\end{array}$} \\
\hline $\begin{array}{l}\text { Restriction of } \\
\text { mouth } \\
\text { opening }\end{array}$ & $35(58.3)$ & 8 & 27 & 0.6227 & 4 & 31 & 0.4367 \\
\hline $\begin{array}{l}\text { Burning } \\
\text { senseation }\end{array}$ & $10(16.7)$ & 2 & 8 & & 2 & 8 & \\
\hline $\begin{array}{l}\text { Blister or } \\
\text { ulcer }\end{array}$ & $14(23.3)$ & 2 & 12 & & 3 & 11 & \\
\hline $\begin{array}{l}\text { Eating } \\
\text { stimulation } \\
\text { pain }\end{array}$ & $21(35)$ & 2 & 19 & & 1 & 20 & \\
\hline
\end{tabular}




\section{Discussions}

Recent reports have shown that the oral mucosa has progressed from normal to OSF clinically and pathologically in some betel nut chewing enthusiasts [26, 27]. However, the molecular mechanism of the initiation and progression of OSF from normal tissue to fibrotic state is still unclear. Earlier studies have demonstrated that the basic pathological process of OSF is the microtrauma caused by the continuous friction of betel nut crude fibers, leading to the infiltration of inflammatory cells in the proximal epithelial area, and the secretion of cytokines. Subsequently, these cytokines act on fibroblasts and promote fibroblasts proliferation and stimulate the production of collagen fibers and ECM in large quantities. To the best of our knowledge, the defective ECM dynamics is the critical procedure of OSF. These classic theories implicate an underlying connection between the pathologic process of OSF and ECM.

MFAP4 is one of the potent members of the ECMs. Current research has shown that MFAP4 expression is correlated with numbers of functions, including coagulation, angiogenesis, tissue growth and remodeling and innate immunity [28-30]. Furthermore, increased MFAP4 protein play an extremely significant role in the development of fibrotic diseases, including heart, liver, joint and kidney fibrosis [10, 13, 14, 16, 25, 31, 32]. However, whether MFAP4 is related to OSF procedure has not been intensively investigated. Our previous research reported that MFAP4 was notably upregulated in OSF tissues by proteomic analysis [5]. Herein, we found that the MFAP4 protein is over-expressed in most samples of OSF compared with that of normal tissue. More importantly, the increase was much more dramatic in the late stage of OSF than in the early stage. Therefore, these results suggested that MFAP4 play an important part in the pathogenesis of OSF and has the latent capacity to be used as a diagnosis and therapy marker of OSF.

In addition, TE, a soluble precursor of elastin and also one of the powerful members of the ECMs, was believed as pro-fibrosis factors. Previous studies have indicated that the expression of TE was significantly increased in asbestos-induced airway fibrosis in rats and in fibrotic tissues such as skin white lines, fine-line scars, hypertrophic scars and keloids, becoming closely correlated with pulmonary fibrosis and skin fibrosis [20-22].Moreover, MFAP4 participate in the assembly of elastin together with TE and the two proteins were both up-regulated in liver fibrosis and cirrhosis, and can serve as a biomarker of liver fibrosis [14, 17, 23]. In liver fibrosis, TE acts directly on fibroblasts and inflammatory cells, and promotes angiogenesis, thus promoting the development of fibrosis [33]. In our study, we observed that the expression of TE was significantly higher in the OSF samples than that in the control samples, being in line with other fibrotic diseases. Moreover, we demonstrate that expression of TE was obviously enhanced in later stage of OSF versus early stage. And then we analyzed the relevance between MFAP4 and TE by the Pearson's correlation test and linear regression, concluded that the expression level of TE was positively associated with MFAP4 protein, indicating that MFAP4 and TE may corporately regulate the development of OSF.

Finally, we assessed the correlation between the expression of MFAP4 and TE and the clinical data in OSF individuals. As shown in Table 1, there is a negative correlation between the MFAP4 and TE expression level and gender, smoking and various clinical features. Nevertheless, statistically remarkable differences were found between the expression of two proteins and chewed areca nut, age and the severity of OSF (TABLE1). The disease tends to 
occur in young and middle-aged people. Consistently, the positive expression rate of MFAP4 and TE in patients less than 40 years old is higher than that of those over 40 years old.

In conclusion, we report here that OSF is associated with increased expression of MFAP4 and TE. Furthermore, the positive expression of MFAP4 and TE proteins increased gradually in early, middle and late OSF tissues, and there was a strong correlation between the expression of MFAP4 and TE in OSF disease. This allows us to conclude that the MFAP4 and TE proteins should be closely relevant to the development of OSF and have potential clinical value acted as novel biomarkers for diagnosis and therapy of OSF.

\section{ETHICS APPROVAL AND CONSENT TO PARTICIPATE}

Informed consent was signed by all study participants, and the research was authorized by the institutional research ethics committee of Xiangya Stomatological Medical College (approval number 20200034).

\section{CONSENT FOR PUBLICATION}

Not applicable

\section{AVAILABILITY OF DATA AND MATERIALS}

The authors agree to open access for this publication.

\section{COMPETING INTERESTS}

The authors declare that they have no competing interests.

\section{FUNDING}

This work was supported by the National Natural Science Foundation of China [81700988] from the Central South university

\section{AUTHOR CONTRIBUTIONS}

Wenqun Gou: conception; design; data acquisition and interpretation; performed all statistical analyses; drafted and critically revised the manuscript. Binjie Liu: data analysis and interpretation; critically revised the manuscript. Hui Feng: conception; design; data acquisition and interpretation; performed all statistical analyses; drafted and critically revised the manuscript. All authors gave their final approval and agree to be accountable for all aspects of the work.

\section{ACKNOWLEDGEMENTS}

We thank Zhigang Yao, Long Li and Gui Liu from the Pathology department of Xiangya Stomatological hospital for helping us confirm pathological diagnosis. We are grateful to Mr. Peng from Hunan Key Laboratory of skin cancer and psoriasis for providing us with experimental technical guidance.

\section{References}

1. Dionne KR, Warnakulasuriya S, Zain RB, Cheong SC. Potentially malignant disorders of the oral cavity: current practice and future directions in the clinic and laboratory. Int $\mathrm{J}$ 
Cancer. 2015; 136: 503 -15.

2. Xie CQ, Feng H, Zhong L, Shi YJ, Wei ZH, Hua YF, et al. Proliferative ability and accumulation of cancer stem cells in oral submucous fibrosis epithelium. Oral Dis. 2020; 26:1255 - 64 .

3. Arora R, Adwani D, Naphade M, Bhagat B, Qureshi AQ. Malignant conversion of oral submucous fibrosis in surgically treated case. J Clin Diagn Res. 2014; 8: 31 - 2.

4. Arakeri G, Rai KK, Hunasgi S, Merkx MAW, Gao S, Brennan PA. Oral submucous fibrosis: An update on current theories of pathogenesis. J Oral Pathol Med. 2017; 46: 406 $-12$.

5. Yuan Y, Huang XH, Feng H F, Liu Y, Xu H, Gong W, et al. Proteomic identification of cyclophilin $\mathrm{A}$ as a potential biomarker and therapeutic target in oral submucous fibrosis. Oncotarget. 2016; 7: 60348 - 65.

6. Kasamatsu S, Hachiya A, Fujimura T, Sriwiriyanont P, Haketa K, Visscher MO, et al. Essential role of microfibrillar-associated protein 4 in human cutaneous homeostasis and in its photoprotection. Sci Rep. 2011: 1:164.

7. Modrego J, López-Farré AJ, Martínez-López I, Muela M, Macaya C, Serrano J, et al. Expression of cytoskeleton and energetic metabolism-related proteins at human abdominal aortic aneurysm sites. J Vasc Surg. 2012; 55: 1124 - 33.

8. de la Cuesta F, Alvarez-Llamas G, Maroto AS, Donado A, Zubiri I, Posada M, et al. A proteomic focus on the alterations occurring at the human atherosclerotic coronary intima. Mol Cell Proteomics. 2011; 10: M110 003517.

9. Pierschbacher MD, Ruoslahti E. Cell attachment activity of fibronectin can be duplicated by small synthetic fragments of the molecule. Nature. 1984; 309: 30 - 3.

10. Pan Z, Yang K, Wang H, Xiao Y, Zhang M, Yu X, et al. MFAP4 deficiency alleviates renal fibrosis through inhibition of NF-kappaB and TGF-beta/Smad signaling pathways. FASEB J. 2020: 34: 14250 - 14263.

11. Madsen BS, Thiele M, Detlefsen S, Sorensen MD, Kjaergaard M, Moller LS, et al. Prediction of liver fibrosis severity in alcoholic liver disease by human microfibrillar-associated protein 4. Liver Int. 2020; 40:1701-12.

12. Wang $\mathrm{HB}$, Huang $\mathrm{R}$, Yang $\mathrm{K}, \mathrm{Xu} \mathrm{M}$, Fan $\mathrm{D}$, Liu $\mathrm{MX}$, et al. Identification of differentially expressed genes and preliminary validations in cardiac pathological remodeling induced by transverse aortic constriction. Int J Mol Med. 2019; 44: 1447 61.

13. Bracht T, Schweinsberg V, Trippler M, Kohl M, Ahrens M, Padden J, et al. Analysis of disease-associated protein expression using quantitative proteomics-fibulin-5 is expressed in association with hepaticfibrosis. J. Proteome Res. 2015; 14: 2278 - 86.

14. Molleken C, Ahrens M, Schlosser A, Dietz J, Eisenacher M, Meyer HE, et al. Direct-acting antivirals-based therapy decreases hepatic fibrosis serum biomarker microfibrillar-associated protein 4 in hepatitis C patients. Clin Mol Hepatol. 2019; 25: 42 -51 .

15. Molleken C, Sitek B, Henkel C, Poschmann G, Sipos B, Stuhler K, et al. Detection of novel biomarkers of liver cirrhosis by proteomic analysis. Hepatology. 2009; 49: 1257 66.

16. Zhang $\mathrm{X}$, Li H, Kou W, Tang $\mathrm{K}$, Zhao $\mathrm{D}$, Zhang $\mathrm{J}$, et al. Increased plasma 
microfibrillar-associated protein 4 is associated with atrial fibrillation and more advanced left atrial remodelling. Arch Med Sci. 2019; 15: 632 - 40.

17. Pilecki B, Holm AT, Schlosser A, Moeller JB, Wohl AP, Zuk AV, et al. Characterization of Microfibrillar-associated Protein 4 (MFAP4) as a Tropoelastin- and Fibrillin-binding Protein Involved in Elastic Fiber Formation. J Biol Chem. 2016; 291: 1103-14.

18. Chen Z, Zhou FL, Zhang SJ, Tian Y, Tian S, Zhang JZ. Modulation of tropoelastin and fibrillin-1 by infrared radiation in human skin in vivo. Photodermatol Photoimmunol Photomed. 2009; 25: 310 - 6.

19. Lockhart-Cairns MP, Newandee H, Thomson J, Weiss AS, Baldock C, Tarakanova A. Transglutaminase-Mediated Cross-Linking of Tropoelastin to Fibrillin Stabilises the Elastin Precursor Prior to Elastic Fibre Assembly. J Mol Biol. 2020; 432: 5736 - 5751.

20. Dai J, Gilks B, Price K, Churg A. Mineral Dusts Directly Induce Epithelial and Interstitial Fibrogenic Mediators and Matrix Components in the Airway Wall. Am J Respir Crit Care Med. 1998; 158:1907 - 13.

21. Janssen R, de Brouwer B, von der Thusen JH, Wouters EFM. Copper as the most likely pathogenic divergence factor between lung fibrosis and emphysema. Med Hypotheses. 2018; 120: 49 - 54.

22. Mendoza-Garcia J, Sebastian A, Alonso-Rasgado T, Bayat A. Ex vivo evaluation of the effect of photodynamic therapy on skin scars and striae distensae. Photodermatol Photoimmunol Photomed. 2015; 31: 239-51.

23. Chen W, Yan XZ, Xu AJ, Sun YM, Wang BQ, Huang T, et al. Dynamics of elastin in liver fibrosis: Accumulates late during progression and degrades slowly in regression. $\mathrm{J}$ Cell Physiol. 2019; 234: 22613 - 22.

24. Ray JG, Smitha T. Oral submucous fibrosis: An update. J Oral Maxillofac Pathol. 2017; 21: $330-331$

25. Madsen B, Thiele M, Detlefsen S, Kjærgaard M, Møller LS, Rasmussen DNB, et al. Human microfibrillar-associated protein 4 expressed in the liver and serum in alcoholic liver disease predicts liver fibrosis severity with accuracy similar to transient elastography and enhanced liver fibrosis test. J Hepatol. 2019; 70: e280.

26. Zhong X, Lu Q, Zhang Q, He Y, Wei W, Wang Y. Oral microbiota alteration associated with oral cancer and areca chewing. Oral Dis. 2020; 27: 226-239.

27. Avinash TML, Anulekha CK, Afroze MM, Prashanth SK, Chatra L, Bhayya H. A correlation between oral mucosal lesions and various quid-chewing habit patterns: A cross-sectional study. J Cancer Res Ther. 2019; 15: 620 - 4.

28. Thomsen T, Schlosser A, Holmskov U, Sorensen GL. Ficolins and FIBCD1: Soluble and membrane bound pattern recognition molecules with acetyl group selectivity. Mol Immunol. 2011; 48: 369 - 81.

29. Toyoshima T, Nishi N, Kusama H, Kobayashi R, Itano T. 36-kDa microfibril-associated glycoprotein (MAGP-36) is an elastin-binding protein increased in chick aortae during development and growth. Exp Cell Res. 2005; 307: 224 - 30.

30. Schlosser A, Pilecki B, Hemstra LE, Karin KJ, Kristmannsdottir GB, Wulf-johansson H, et al. MFAP4 Promotes Vascular Smooth Muscle Migration, Proliferation and Accelerates Neointima Formation. Arterioscler Thromb Vasc Biol. 2016; 36: 122 - 33.

31. Melenovsky V, Cervenka L, Viklicky O, Franekova J, Havlenova T, Behounek M, et al. 
Kidney Response to Heart Failure: Proteomic Analysis of Cardiorenal Syndrome. Kidney Blood Press Res. 2018; 43: 1437 - 50.

32. Christensen AF, Sorensen GL, Junker K, Revald P, Varnum C, Issa SF, et al. Site-specific absence of microfibrillar-associated protein 4 (MFAP4) from the internal elastic membrane of arterioles in the rheumatoid arthritis synovial membrane: an immunohistochemical study in patients with advanced rheumatoid arthritis versus osteoarthritis. APMIS. 2019; 127: 588 - 93.

33. Nakayama H, Itoh H, Kunita S, Kuroda N, Hiroi M, Matsuura H, et al. Presence of perivenular elastic fibers in nonalcoholic steatohepatitis fibrosis stage III. Histo Histopathol. 2008; 23: 407 - 9. 